\title{
On a Partial Boundary Value Condition of a Porous Medium Equation with Exponent Variable
}

\author{
Huashui Zhan iD ${ }^{1,2}$ \\ ${ }^{1}$ School of Applied Mathematics, Xiamen University of Technology, Xiamen 361024, China \\ ${ }^{2}$ Fujian Engineering and Research Center of Rural Sewage Treatment and Water Safety, Xiamen 361024, China
}

Correspondence should be addressed to Huashui Zhan; huashuizhan@163.com

Received 9 August 2019; Accepted 11 February 2020; Published 14 March 2020

Academic Editor: Genni Fragnelli

Copyright (c) 2020 Huashui Zhan. This is an open access article distributed under the Creative Commons Attribution License, which permits unrestricted use, distribution, and reproduction in any medium, provided the original work is properly cited.

The initial-boundary value problem of a porous medium equation with a variable exponent is considered. Both the diffusion coefficient $a(x, t)$ and the variable exponent $p(x, t)$ depend on the time variable $t$, and this makes the partial boundary value condition not be expressed as the usual Dirichlet boundary value condition. In other words, the partial boundary value condition matching up with the equation is based on a submanifold of $\partial \Omega \times(0, T)$. By this innovation, the stability of weak solutions is proved.

\section{Introduction}

The porous medium equation with a constant exponent is widely used to model several real-life problems, and it has been extensively studied, one can refer to the survey books [1-6] and the references therein. The dynamics system of a partially nonlocal and inhomogeneous nonlinear medium has been considered in [7-9]. The case where the exponent of nonlinearity is not constant was proposed by Antontsev and Shmarev in [10], where the existence, uniqueness, and some properties of the solution in a bounded fixed domain were researched. By using the Galerkin finite element method, Duque et al. [11] proved the convergence of a fully discrete solution for this problem in a fixed domain. Based on one of the properties proved in [11] that the solution is with the finite speed of propagation, Duque et al. [12] considered the free boundary problem by using the moving mesh method to the porous medium. However, the moving mesh method was first introduced by Huang and Russell in [13].

In this paper, we consider the initial-boundary value problem of a generalized porous medium equation with a variable exponent:

$$
\begin{array}{r}
u_{t}=\operatorname{div}\left(a(x, t)|u|^{m(x, t)} \nabla u\right)+\sum_{i=1}^{N} \frac{\partial b_{i}\left(u^{m(x, t)+1}\right)}{\partial x_{i}}, \\
\quad(x, t) \in Q_{T}=\Omega \times(0, T),
\end{array}
$$

where $m(x, t)>0$ is a $C^{1}\left(\overline{Q_{T}}\right)$ function, $a(x, t) \geq 0$ is a $C^{1}\left(\overline{Q_{T}}\right)$ function, $b_{i}(s) \in C^{1}(\mathbb{R})$, and $\Omega \subset \mathbb{R}^{N}$ is a bounded domain with a smooth boundary $\partial \Omega$.

Equation (1) is a special case of the reaction-diffusion equation:

$$
u_{t}=\operatorname{div}(a(u, x, t) \nabla u)+\operatorname{div}(\vec{b}(u, x, t))
$$

Because $a(\cdot, x, t)$ may degenerate on the boundary, how to impose a suitable boundary value condition to study the well posedness of weak solutions to equation (2) has attracted extensive attention and has been widely studied for a long time. In some details, though the initial value

$$
u(x, 0)=u_{0}(x), \quad x \in \Omega,
$$

is always imposed, the Dirichlet boundary condition

$$
u(x, t)=0,(x, t) \in \partial \Omega \times(0, T),
$$


may not be imposed or be imposed in a weaker sense than the traditional trace. One can refer to the references [14-19] for the details.

Naturally, besides the porous medium equation with variable exponents, the so-called electrorheological fluid equations with the form

$$
u_{t}=\operatorname{div}\left(|\nabla u|^{p(x)-2} \nabla u\right)+f(x, t, u, \nabla u),
$$

have been brought to the forefront by many more scholars. Since the beginning of this century, there are a great deal of papers devoting to the well-posedness problem, the intrinsic Harnack inequalities, the long-time behavior, and the Hölder regularity of weak solutions, one can refer to the literatures [20-31] and the references therein.

$$
\begin{aligned}
& \text { If } m(x, t)=m(x) \text {, then } a(x, t)=a(x) \text { satisfies } \\
& \qquad \begin{array}{c}
a(x)>0, x \in \Omega, a(x)=0, x \in \partial \Omega, \\
0 \leq u_{0} \in L^{\infty}(\Omega), \\
\sqrt{a(x)} \nabla u_{0}^{m(x)} \in L^{2}(\Omega) .
\end{array}
\end{aligned}
$$

The existence and the stability of weak solutions to equation

$$
\begin{array}{r}
u_{t}=\operatorname{div}\left(a(x)|u|^{m(x)} \nabla u\right)+\sum_{i=1}^{N} \frac{\partial b_{i}\left(u^{m(x)+1}\right)}{\partial x_{i}}, \\
(x, t) \in \Omega \times(0, T),
\end{array}
$$

has been studied in [32]. It is found that the degeneracy of $a(x)$ on boundary (6) may replace the usual boundary value condition (4). In other words, if $a(x)$ satisfies (6), the stability of weak solutions may be proved without the usual boundary value condition (4).

In this paper, we will use some ideas of [32] to study the well posedness of weak solutions to equation (1). Because both $a(x, t)$ and $p(x, t)$ are dependent on the time variable $t$, the problem becomes more difficult and the question of existence of such solutions is still open for equation (8), as well as for evolution $p$-Laplace equation with the exponent $p$ depending on $t$ [22]. Instead of condition (6), we only assume that $a(x, t) \geq 0$. By this assumption, we find out a partial boundary value condition matching up with the equation. Moreover, because both $a(x, t)$ and $p(x, t)$ are dependent on the time variable $t$, the partial boundary value condition cannot be expressed as the usual Dirichlet boundary value condition. In other words, the partial boundary value condition is based on a submanifold of $\partial \Omega \times(0, T)$. By this innovation, the stability of weak solutions is proved.

\section{The Partial Boundary Value Condition and the Main Results}

For any given $t \in[0, T)$ and small enough $\lambda>0$, we set

$$
\begin{aligned}
\Omega_{\lambda t} & =\{x \in \Omega: a(x, t)>\lambda\}, \\
\Omega_{\lambda i 1 t} & =\left\{x \in \Omega \backslash \Omega_{\lambda t}: a_{x_{i}}<0\right\}, \\
\Sigma_{1 i t} & =\lim _{\lambda \longrightarrow 0} \Omega_{\lambda i 1 t}, \quad i=1,2, \ldots, N, \\
\Omega_{\lambda i 2 t} & =\left\{x \in \Omega \backslash \Omega_{\lambda t}: a_{x_{i}} \geq 0\right\}, \\
\Sigma_{2 i t} & =\lim _{\lambda \longrightarrow 0} \Omega_{\lambda i 2 t}, \quad i=1,2, \ldots, N .
\end{aligned}
$$

The most important and essential improvement is that instead of the usual boundary value condition (4), the stability of weak solutions is proved based on a partial boundary value condition:

$$
u(x, t)=0,(x, t) \in \Sigma_{1} \cup \Sigma_{2},
$$

where

$$
\Sigma_{2}=\{x \in \partial \Omega: a(x, t) \neq 0\},
$$

and for any given $i \in\{1,2, \ldots, N\}$, if $b_{i}^{\prime}(s) \geq 0$,

$$
\Sigma_{1}=\left\{\bigcup_{i=1}^{N} \Sigma_{1 i t} \times(0, T)\right\}
$$

and if $b_{i}^{\prime}(s) \leq 0$,

$$
\Sigma_{1}=\left\{\bigcup_{i=1}^{N} \Sigma_{2 i t} \times(0, T)\right\} .
$$

Thus, if for every $i \in\{1,2, \ldots, N\}$, either $b_{i}^{\prime}(s) \geq 0$ or $b_{i}^{\prime}(s) \leq 0$, then one can deduce an expression $\Sigma_{1}$ from the above discussion. For example, $b_{i}(s) \geq 0$ when $1 \leq i \leq k$ and $b_{i}^{\prime}(s) \leq 0$ when $k+1 \leq i \leq N$; then,

$$
\Sigma_{1}=\left\{\bigcup_{i=1}^{k} \Sigma_{1 i t} \times(0, T)\right\} \cup\left\{\bigcup_{i=k+1}^{N} \Sigma_{2 i t} \times(0, T)\right\} .
$$

The most characteristic out of the ordinary is that $\Sigma_{1}$ or $\Sigma_{2}$ is just a submanifold of $\partial \Omega \times(0, T)$, and it cannot be expressed as a cylinder with the form $\Gamma \times(0, T)$ and $\Gamma \subset \partial \Omega$.

Definition 1. If $u(x, t) \geq 0$ and satisfies

$$
\begin{array}{r}
u \in L^{\infty}\left(Q_{T}\right), \\
\sqrt{a(x, 0)}|u|^{m(x, 0)}|\nabla u| \in L^{\infty}\left(0, T ; L^{2}(\Omega)\right),
\end{array}
$$

and for $\forall \varphi \in C_{0}^{1}\left(Q_{T}\right)$,

$$
\begin{array}{r}
\iint_{Q_{T}}\left(-\frac{\partial \varphi}{\partial t} u+a(x, t)|u|^{m(x, t)} \nabla u \nabla \varphi\right) \mathrm{d} x \mathrm{~d} t \\
+\sum_{i=1}^{N} \iint_{Q_{T}} b_{i}\left(u^{m(x, t)+1}\right) \varphi_{x_{i}}(x, t) \mathrm{d} x \mathrm{~d} t=0,
\end{array}
$$

and then $u(x, t)$ is said to be a weak solution of equation (1) with the initial value $(3)$ in the sense 


$$
\lim _{t \rightarrow 0} \int_{\Omega}\left|u(x, t)-u_{0}(x)\right| \mathrm{d} x=0 .
$$

Moreover, if $u(x, t)$ satisfies (4) or (3) in the sense of the trace in addition, then it is said to be a weak solution of the initial-boundary value problem of equation (1).

Theorem 1. If $m(x, t)>0$ is a $C^{1}\left(\overline{Q_{T}}\right)$ function, $b_{i}(s)$ satisfies

$$
\left|b_{i}\left(s_{1}\right)-b_{i}\left(s_{2}\right)\right| \leq c\left|s_{1}-s_{2}\right|, \quad i=1,2, \ldots, N .
$$

If $u_{0}(x) \geq 0$ satisfies (16), then equation (1) with initial value (3) has a nonnegative solution.

Theorem 2. Let $m(x, t)>0$ be a $C^{1}\left(\overline{Q_{T}}\right)$ function, then $b_{i}(s) \in C^{1}(\mathbb{R})$ satisfy $(19)$ :

$$
\int_{\Omega} a^{-1}(x, t) \mathrm{d} x \leq c(T) .
$$

Then, the initial-boundary value problem (1), (3), and (4) has a uniqueness solution.

Theorem 3. Let $u(x, t)$ and $v(x, t)$ be two solutions of equation (1) with the initial values $u_{0}(x)$ and $v_{0}(x)$, respectively, and with a partial boundary value condition

$$
u(x, t)=v(x, t)=0, \quad(x, t) \in \Sigma_{1} \cup \Sigma_{2} .
$$

It is supposed that, for every $i \in\{1,2, \ldots, N\}$, either $b_{i}^{\prime}(s) \geq 0$ or $b_{i}^{\prime}(s) \leq 0, a(x, t)$ satisfies

$$
\frac{1}{\lambda}\left(\int_{\Omega \backslash \Omega_{\lambda t}} a(x, t)|\nabla a|^{2} \mathrm{~d} x\right)^{1 / 2} \leq c(T),
$$

and $u(x, t)$ and $v(x, t)$ satisfy

$$
\begin{aligned}
& \int_{\Omega} a(x, t)[1+(m(x, t)+1) \log u]^{2}|\nabla m|^{2} \mathrm{~d} x \leq c(T), \\
& \int_{\Omega} a(x, t)[1+(m(x, t)+1) \log v]^{2}|\nabla m|^{2} \mathrm{~d} x \leq c(T) .
\end{aligned}
$$

Then,

$$
\int_{\Omega}|u(x, t)-v(x, t)| \mathrm{d} x \leq c(T) \int_{\Omega}\left|u_{0}(x)-v_{0}(x)\right| \mathrm{d} x .
$$

Hereinafter, the constant $c(T)$ represents a constant which depends on $T$.

At the end of this section, we would like to suggest that if $m(x, t)=m$ is a constant, then condition (22) in Theorem 3 is naturally true.

\section{The Proof of Theorem 1}

First, we suppose that $u_{0} \in C_{0}^{\infty}(\Omega)$ and $0 \leq u_{0} \leq M$ and consider the following regularized problem:

$$
\begin{cases}u_{n t}=\operatorname{div}\left(\left(a(x, t)+\frac{1}{n}\right)\left(\left|u_{n}\right|^{m(x, t)}+\frac{1}{n}\right) \nabla u\right)+\sum_{i=1}^{N} \frac{\partial b_{i}\left(u_{n}^{m(x, t)+1}\right)}{\partial x_{i}},(x, t), & (x, t) \in Q_{T}, \\ u_{n}(x, t)=\frac{1}{n}, & (x, t) \in \partial \Omega \times(0, T), \\ u_{n}(x, 0)=u_{0 n}(x)=u_{0}(x)+\frac{1}{n}, & x \in \Omega .\end{cases}
$$

According to the standard parabolic equation theory, there is a weak solution $u_{n} \in L^{\infty}\left(Q_{T}\right)$ satisfying

$$
\begin{aligned}
& \left(a(x, t)+\frac{1}{n}\right)^{1 / 2}\left(\left|u_{n}\right|^{m(x, t)}+\frac{1}{n}\right)^{1 / 2} \nabla u \in L^{2}\left(Q_{T}\right), \\
& \frac{1}{n} \leq u_{n}(x, t) \leq\left\|u_{0}\right\|_{L^{\infty}(\Omega)}+\frac{1}{n}, \quad(x, t) \in Q_{T} .
\end{aligned}
$$

Moreover, by comparison theorem, we clearly have

$$
u_{n+1}(x, t) \leq u_{n}(x, t) \text {, }
$$

which yields

$$
\begin{aligned}
& u(x, t)=\lim _{n \longrightarrow \infty} u_{n}(x, t), \\
& |u(x, t)| \leq M+1 .
\end{aligned}
$$

In what follows, we are able to prove that the limit function $u$ is a weak solution of (1) with the initial value (3).

Multiplying both sides of the first equation in (25) by $\phi=(m(x, t)+2)\left(u_{n}^{m(x, t)+1}-(1 / n)^{m(x, t)+1}\right)$ and integrating it over $Q_{T}$, we have 


$$
\begin{aligned}
& \iint_{Q_{T}} u_{n t}\left(u_{n}^{m(x)+1}-\left(\frac{1}{n}\right)^{m(x, t)+1}\right)(m(x, t)+2) \mathrm{d} x \mathrm{~d} t \\
& =\iint_{Q_{T}} \operatorname{div}\left[\left(a(x, t)+\frac{1}{n}\right)\left(\left|u_{n}\right|^{m(x, t)}+\frac{1}{n}\right) \nabla u\right]\left(u_{n}^{m(x, t)+1}-\frac{1}{n^{m(x, t)+1}}\right)(m(x, t)+2) \mathrm{d} x \mathrm{~d} t \\
& \quad+\sum_{i=1}^{N} \iint_{Q_{T}} \frac{\partial b_{i}\left(u_{n}^{m(x, t)+1}\right)}{\partial x_{i}}\left(u_{n}^{m(x, t)+1}-\left(\frac{1}{n}\right)^{m(x)+1}\right)(m(x, t)+2) \mathrm{d} x \mathrm{~d} t .
\end{aligned}
$$

For the left-hand side of (29),

$$
\begin{aligned}
& \iint_{Q_{t}} u_{n t}\left(u_{n}^{m(x, t)+1}-\left(\frac{1}{n}\right)^{m(x, t)+1}\right)(m(x, t)+2) \mathrm{d} x \mathrm{~d} t \\
& =\int_{\Omega}\left[u_{n}^{m(x, t)+2}(x, t)-u_{n}^{m(x)+2}(x, 0)\right] \mathrm{d} x \\
& \quad-\int_{\Omega}\left(\frac{1}{n}\right)^{m(x, t)+1}\left[u_{n}(x, t)-u_{n}(x, 0)\right](m(x, t)+2) \mathrm{d} x \\
& +\iint_{Q_{T}} u_{n} \frac{\partial}{\partial t}\left[\left(\frac{1}{n}\right)^{m(x, t)+1}(m(x, t)+2)\right] \mathrm{d} x \mathrm{~d} t .
\end{aligned}
$$

For the first term of the right-hand side of (29), because

$$
\begin{aligned}
& \lim _{n \longrightarrow \infty}\left(a(x, t)+\frac{1}{n}\right)\left(\left|u_{n}\right|^{m(x, t)}+\frac{1}{n}\right)\left[(m(x, t)+1) u_{n}^{m(x, t)}\right]^{-1}|\nabla m|^{2} \\
& \quad \cdot\left|\log u_{n} u_{n}^{m(x, t)+1}-\left(\frac{1}{n}\right)^{m(x, t)+1} \log n\right| \\
& \quad=a(x, t)(m(x, t)+1)^{-1}|\nabla m(x, t)|^{2} u^{m(x, t)+1}|\log u|<\infty, \\
& \left.\iint_{Q_{T}}\left(a(x, t)+\frac{1}{n}\right)\left(\left|u_{n}\right|^{m(x, t)}+\frac{1}{n}\right)\left(u_{n}^{m(x, t)+1}-\frac{1}{n^{m(x, t)}+1}\right) \nabla m\right|^{2} \mathrm{~d} x \mathrm{~d} t<\infty,
\end{aligned}
$$

by a complicated calculation and using the Young inequality,

we can deduce that

$$
\begin{gathered}
\iint_{Q_{T}} \operatorname{div}\left[\left(a(x, t)+\frac{1}{n}\right)\left(\left|u_{n}\right|^{m(x, t)}+\frac{1}{n}\right) \nabla u_{n}\right]\left(u_{n}^{m(x, t)+1}-\frac{1}{n^{m(x, t)+1}}\right)(m(x, t)+2) \mathrm{d} x \mathrm{~d} t \\
\leq-c \iint_{Q_{T}}\left(a(x, t)+\frac{1}{n}\right)\left(\left|u_{n}\right|^{m(x, t)}+\frac{1}{n}\right)(m(x, t)+1) u_{n}^{m(x, t)}\left|\nabla u_{n}\right|^{2} \mathrm{~d} x \mathrm{~d} t+c .
\end{gathered}
$$

For the second term of the right-hand side of (29), because 


$$
\begin{aligned}
& \iint_{Q_{T}} \frac{\partial b_{i}\left(u_{n}^{m(x, t)+1}\right)}{\partial x_{i}} u_{n}^{m(x, t)+1}(m(x, t)+2) \mathrm{d} x \mathrm{~d} t \\
& =-\iint_{Q_{T}} b_{i}\left(u_{n}^{m(x, t)+1}\right) \frac{\partial}{\partial x_{i}}\left(u_{n}^{m(x)+1}(m(x, t)+2)\right) \mathrm{d} x \mathrm{~d} t \\
& =-\iint_{Q_{T}}\left[\frac{\partial}{\partial x_{i}} \int_{(1 / n)^{m(x, t)+1}}^{u_{n}^{m(x, t)+1}} b_{i}(s) \mathrm{d} s+b_{i}\left(n^{-m(x, t)-1}\right)\left(\frac{1}{n}\right)^{m(x, t)+1} \log n m_{x_{i}}(x, t)\right](m(x, t)+2) \mathrm{d} x \mathrm{~d} t \\
& \quad-\iint_{Q_{T}} b_{i}\left(u_{n}^{m(x, t)+1}\right) u_{n}^{m(x, t)+1} m_{x_{i}} \mathrm{~d} x \mathrm{~d} t \\
& =-\iint_{Q_{T}} b_{i}\left(n^{-m(x, t)-1}\right)\left(\frac{1}{n}\right)^{m(x, t)+1} \log n m_{x_{i}}(x, t) \mathrm{d} x \mathrm{~d} t-\iint_{Q_{T}} b_{i}\left(u_{n}^{m(x, t)+1}\right) u_{n}^{m(x, t)+1} m_{x_{i}} \mathrm{~d} x \mathrm{~d} t,
\end{aligned}
$$

we can deduce that

$$
\left|\sum_{i=1}^{N} \iint_{Q_{T}} \frac{\partial b_{i}\left(u_{n}^{m(x, t)+1}\right)}{\partial x_{i}}\left(u_{n}^{m(x, t)+1}-\left(\frac{1}{n}\right)^{m(x, t)+1}\right) \mathrm{d} x \mathrm{~d} t\right| \leq c .
$$

From (28)-(34), we extrapolate

$$
\iint_{Q_{T}}\left(a(x, t)+\frac{1}{n}\right)\left(\left|u_{n}\right|^{m(x, t)}+\frac{1}{n}\right) u_{n}^{m(x, t)}\left|\nabla u_{n}\right|^{2} \mathrm{~d} x \mathrm{~d} t \leq c .
$$

Accordingly, there is $\vec{\zeta} \in L^{2}\left(Q_{T}\right)$ such that

$$
\left[\left(a(x, t)+\frac{1}{n}\right)\left(\left|u_{n}\right|^{m(x, t)}+\frac{1}{n}\right) u_{n}^{m(x, t)}\right]^{1 / 2} \nabla u_{n}-\vec{\zeta},
$$

weakly in $L^{2}\left(Q_{T}\right)$. We now can prove

$$
\vec{\zeta}=a(x, t)^{1 / 2}|u|^{m(x, t)} \nabla u
$$

as in a similar way as that in [32].

The last but not the least, by that $b_{i} \in C^{1}(\mathbb{R})$, using (27), we have

$$
\lim _{n \longrightarrow \infty} b_{i}\left(u_{n}^{m(x)+1}\right)=b_{i}\left(u^{m(x)+1}\right) .
$$

Letting $n \longrightarrow \infty$ in (30), by (37), (38), and (39), we know $u(x, t)$ satisfies $(18)$.

Secondly, if $u_{0}$ satisfies only (16), we should consider equation (9) with the initial value $u_{0 \varepsilon}$ which is the mollified function of $u_{0}$, from the above that there is a weak solution $u_{\varepsilon}$ satisfying (18). Letting $\varepsilon \longrightarrow 0$, the limit function $u(x, t)$ is a solution of (1) satisfying (17) and (18), but generally is not continuous at $t=0$ as in the case $u_{0} \in C_{0}^{\infty}(\Omega)$.

Thirdly, the initial value (4) can be proved in a similar way as that when $m(x, t)=m-1$ is a constant, one can refer to [5] for the details. Thus, $u$ is a solution of equation (1) with the initial value (4). Thus, Theorem 1 is proved.

\section{The Proof of Theorem 2}

One can see that, in Definition 1, there is not any definition on the general derivative $u_{t}$. At the beginning of this section, we first answer this question.

For any $t \in[0, T)$, the Banach space $V_{t}(\Omega)$ is defined by

$$
\begin{aligned}
V_{t}(\Omega)= & \left\{u(x, t): u(x, t) \in L^{2}(\Omega) \cap W_{0}^{1,1}(\Omega),\right. \\
& \left.\cdot|\nabla u(x, t)|^{2} \in L^{1}(\Omega)\right\}, \\
\|u\|_{V_{t}(\Omega)}= & \|u\|_{2, \Omega}+\|\nabla u\|_{2, \Omega},
\end{aligned}
$$

and $V_{t}^{\prime}(\Omega)$ is denoted as its dual space. The Banach space $W\left(Q_{T}\right)$ is defined by

$$
\begin{aligned}
W\left(Q_{T}\right) & =\left\{u:\left.[0, T] \longrightarrow V_{t}(\Omega)\left|u \in L^{2}\left(Q_{T}\right),\right| \nabla u\right|^{2}\right. \\
& \left.\in L^{1}\left(Q_{T}\right), u=0 \text { on } \Gamma=\partial \Omega\right\}, \\
\|u\|_{W\left(Q_{T}\right)} & =\|\nabla u\|_{2, Q_{T}}+\|u\|_{2, Q_{T}},
\end{aligned}
$$

and $W^{\prime}\left(Q_{T}\right)$ is denoted as its dual space. From [21], we have

$$
w \in W^{\prime}\left(Q_{T}\right) \Leftrightarrow\left\{\begin{array}{l}
w=w_{0}+\sum_{i=1}^{N} D_{i} w_{i}, \quad w_{0} \in L^{2}\left(Q_{T}\right), w_{i} \in L^{2}\left(Q_{T}\right), \\
\forall \phi \in W\left(Q_{T}\right), \ll w, \phi \gg=\iint_{Q_{T}}\left(w_{0} \phi+\sum_{i}^{N} w_{i} D_{i} \phi\right) \mathrm{d} x \mathrm{~d} t .
\end{array}\right.
$$

It is easy to prove the following lemmas, so we omit the details here.
Lemma 1. If $u(x, t)$ is a weak solution of equation (1) with the initial value (3), then $u_{t} \in W^{\prime}\left(Q_{T}\right)$. 
Lemma 2. Suppose that $u \in W\left(Q_{T}\right)$ and $u_{t} \in W^{\prime}\left(Q_{T}\right)$. For any continuous function $h(s)$, let $H(s)=\int_{0}^{s} h(s) \mathrm{d} s$. For a.e. $t_{1}, t_{2} \in(0, T)$, there holds

$$
\int_{t_{1}}^{t_{2}} \int_{\Omega} h(u) u_{t} \mathrm{~d} x \mathrm{~d} t=\left[\int_{\Omega}\left(H(u)\left(x, t_{2}\right)-H(u)\left(x, t_{1}\right)\right) \mathrm{d} x\right] .
$$

Lemma 3. If $\int_{\Omega} a(x, t)^{-1} \mathrm{~d} x \leq c(T)$, then $\int_{\Omega}\left|\nabla u^{m(x, t)+1}\right|$ $\mathrm{d} x \leq c(T)$. So, the weak solution of equation (1) $u(x, t)$ can be defined as the homogeneous boundary value condition $\left.u\right|_{\partial \Omega}=$ 0 in the sense of the trace.

Theorem 4. Let $u(x, t)$ and $v(x, t)$ be two solutions of equation (1) with the initial values $u_{0}(x)$ and $v_{0}(x)$ respectively, and with

$$
u(x, t)=v(x, t)=0, \quad(x, t) \in \partial \Omega \times(0, T) .
$$

$$
\begin{aligned}
& \text { If } \int_{\Omega} a(x, t)^{-1}(x) \mathrm{d} x \leq c(T), \text { then } \\
& \int_{\Omega}|u(x, t)-v(x, t)| \leq c \int_{\Omega}\left|u_{0}(x)-v_{0}(x)\right| \mathrm{d} x .
\end{aligned}
$$

Proof. For any given positive integer $n$, let $g_{n}(s)=\int_{0}^{s} h_{n}(\tau) \mathrm{d} \tau$ and $h_{n}(s)=2 n(1-n|s|)_{+} . \quad$ Then, $h_{n}(s) \in C(\mathbb{R})$, and we have

$$
\begin{aligned}
& \lim _{n \longrightarrow \infty} g_{n}(s)=\operatorname{sgn} s, \\
& \lim _{n \longrightarrow \infty} s g_{n}^{\prime}(s)=0 .
\end{aligned}
$$

Because $u(x, t)=v(x, t)=0$ on the boundary $\partial \Omega \times[0, T)$, we choose $g_{n}\left(u^{m(x, t)+1}-v^{m(x, t)+1}\right)$ as the test function and integrate over $Q_{t}=\Omega \times(0, t)$. Then,

$$
\begin{aligned}
& \int_{0}^{t} \int_{\Omega} g_{n}\left(u^{m(x, s)+1}-v^{m(x, s)+1}\right) \frac{\partial(u-v)}{\partial t} \mathrm{~d} x \mathrm{~d} s \\
& \quad+\iint_{Q_{t}} \frac{a(x, s)}{m(x, s)+1}\left|\nabla u^{m(x, s)+1}-\nabla v^{m(x, s)+1}\right|^{2} h_{n}\left(u^{m(x, s)+1}-v^{m(x, s)+1}\right) \mathrm{d} x \mathrm{~d} s \\
& \quad-\iint_{Q_{t}} \frac{a(x, s)}{m(x, s)+1}\left(u^{m(x, s)+1} \log u-v^{m(x, s)+1} \log u\right) \\
& \quad \cdot h_{n}\left(u^{m(x, s)+1}-v^{m(x, s)+1}\right) \nabla m \nabla\left(u^{m(x, s)+1}-v^{m(x, s)+1}\right) \mathrm{d} x \mathrm{~d} s \\
& \quad+\sum_{i=1}^{N} \iint_{Q_{t}}\left[b_{i}\left(u^{m(x, s)+1}\right)-b_{i}\left(v^{m(x, s)+1}\right)\right]\left(u^{m(x, s)+1}-v^{m(x, s)+1}\right)_{x_{i}} \cdot h_{n}\left(u^{m(x, s)+1}-v^{m(x, s)+1}\right) \mathrm{d} x \mathrm{~d} s=0 .
\end{aligned}
$$

Let us analyse every term in (47). In the first place,

$$
\begin{aligned}
& \iint_{Q_{t}} \frac{a(x, s)}{m(x, s)+1}\left|\nabla u^{m(x, s)+1}-\nabla v^{m(x, s)+1}\right|^{2} h_{n}\left(u^{m(x, s)+1}-v^{m(x, s)+1}\right) \mathrm{d} x \mathrm{~d} t \geq 0, \\
& -\iint_{Q_{t}} \frac{a(x, s)}{m(x, s)+1}\left(u^{m(x, s)+1} \log u-v^{m(x, s)+1} \log u\right) \\
& \cdot h_{n}\left(u^{m(x, s)+1}-v^{m(x, s)+1}\right) \nabla m \nabla\left(u^{m(x, s)+1}-v^{m(x, s)+1}\right) \mathrm{d} x \mathrm{~d} s \\
& \geq-\frac{1}{2} \iint_{Q_{t}} \frac{a(x, s)}{m(x, s)+1}\left|\nabla u^{m(x, s)+1}-\nabla v^{m(x, s)+1}\right|^{2} h_{n}\left(u^{m(x, s)+1}-v^{m(x, s)+1}\right) \mathrm{d} x \mathrm{~d} s \\
& -\frac{1}{2} \iint_{Q_{t}} \frac{a(x, s)}{m(x, s)+1}\left[\left(u^{m(x, s)+1} \log u-v^{m(x, s)+1} \log u\right) h_{n}\left(u^{m(x, s)+1}-v^{m(x, s)+1}\right)\right]^{2} \mathrm{~d} x \mathrm{~d} s .
\end{aligned}
$$

In the second place, we deal with the fourth term on the left-hand side of (47). For any given $t \in[0, T)$, we set

$$
\begin{aligned}
& D_{n t}=\left\{\Omega:\left|u^{m(x, t)+1}-v^{m(x, t)+1}\right|<\frac{1}{n}\right\}, \\
& D_{0 t}=\{x \in \Omega:|u-v|=0\} .
\end{aligned}
$$


Clearly,

Based on these denotations, we have

$$
\lim _{n \longrightarrow \infty} D_{n t}=D_{0 t}
$$

$$
\begin{aligned}
\mid \int_{0}^{t} \int_{\Omega} & {\left[b_{i}\left(u^{m(x, s)+1}\right)-b_{i}\left(v^{m(x, s)+1}\right)\right]\left(u^{m(x, s)+1}-v^{m(x, s)+1}\right)_{x_{i}} h_{n}\left(u^{m(x, s)+1}-v^{m(x, s)+1}\right) \mathrm{d} x \mathrm{~d} s \mid } \\
= & \mid \int_{0}^{t} \int_{D_{n s}}\left[b_{i}\left(u^{m(x, s)+1}\right)-b_{i}\left(v^{m(x, s)+1}\right)\right] \\
& \cdot h_{n}\left(u^{m(x, s)+1}-v^{m(x, s)+1}\right)\left(u^{m(x, s)+1}-v^{m(x, s)+1}\right)_{x_{i}} \mathrm{~d} x \mathrm{~d} s \mid \\
\leq & c \int_{0}^{t} \int_{D_{n s}}\left|\frac{b_{i}\left(u^{m(x, s)+1}\right)-b_{i}\left(v^{m(x, s)+1}\right)}{u^{m(x, s)+1}-v^{m(x, s)+1}}\right|\left|\left(u^{m(x, s)+1}-v^{m(x, s)+1}\right)_{x_{i}}\right| \mathrm{d} x \mathrm{~d} s \\
= & c \int_{0}^{t} \int_{D_{n s}}\left|a(x, s)^{-(1 / 2)} \frac{b_{i}\left(u^{m(x, s)+1}\right)-b_{i}\left(v^{m(x, s)+1}\right)}{u^{m(x, s)+1}-v^{m(x, s)+1}}\right|\left|a(x, s)\left(u^{m(x, s)+1}-v^{m(x, s)+1}\right)_{x_{i}}\right| \mathrm{d} x \mathrm{~d} s \\
\leq c & {\left[\int_{0}^{s} \int_{D_{n s}}\left(a(x, s)^{-(1 / 2)} \frac{b_{i}\left(u^{m(x, s)+1}\right)-b_{i}\left(v^{m(x, s)+1}\right)}{u^{m(x, s)+1}-v^{m(x, s)+1}}\right)^{2} \mathrm{~d} x\right]^{1 / 2} } \\
& \cdot\left[\int_{0}^{t} \int_{D_{n s}} a(x, t)\left|\nabla\left(u^{m(x, s)+1}-v^{m(x, s)+1}\right)\right|^{2} \mathrm{~d} x \mathrm{~d} s\right]^{1 / 2} \cdot
\end{aligned}
$$

Because $\int_{\Omega} a(x, s)^{-1}(x) \mathrm{d} x \leq c(T)$,

$$
\begin{aligned}
& \int_{0}^{t} \int_{D_{n s}}\left(a(x, s)^{-(1 / 2)} \frac{b_{i}\left(u^{m(x, s)+1}\right)-b_{i}\left(v^{m(x, s)+1}\right)}{u^{m(x, s)+1}-v^{m(x, s)+1}}\right)^{2} \mathrm{~d} x \mathrm{~d} s \\
& \quad \leq c \int_{D_{n s}} a(x, s)^{-1} \mathrm{~d} x \mathrm{~d} s \\
& \quad \leq c(T) .
\end{aligned}
$$

If $D_{0 s}=\left\{x \in \Omega:\left|u^{m(x, s)+1}-v^{m(x, s)+1}\right|=0\right\}$ has 0 measure, then

$$
\lim _{n \longrightarrow \infty} \int_{0}^{t} \int_{D_{n s}} a(x, s)^{-1} \mathrm{~d} x=\int_{0}^{t} \int_{D_{0 s}} a(x, s)^{-1} \mathrm{~d} x \mathrm{~d} s=0 .
$$

If $D_{0 s}=\left\{x \in \Omega:\left|u^{m(x, s)+1}-v^{m(x, s)+1}\right|=0\right\}$ has a positive measure, then

$$
\begin{aligned}
\lim _{n \rightarrow \infty} & \int_{0}^{t} \int_{D_{n s}} a(x, s)\left|\nabla\left(u^{m(x, s)+1}-v^{m(x, s)+1}\right)\right|^{2} \mathrm{~d} x \mathrm{~d} s \\
& =\int_{0}^{t} \int_{D_{0 s}} a(x, s)\left|\nabla\left(u^{m(x, s)+1}-v^{m(x, s)+1}\right)\right|^{2} \mathrm{~d} x \mathrm{~d} s \\
& =0 .
\end{aligned}
$$

Thus, in both cases, we always have

$$
\begin{aligned}
\lim _{n \longrightarrow \infty} & \int_{0}^{t} \int_{\Omega}\left(b_{i}\left(u^{m(x, s)+1}\right)-b_{i}\left(v^{m(x, s)+1}\right)\right) h_{n} \\
\cdot & \left(u^{m(x, s)+1}-v^{m(x, s)+1}\right) \\
\cdot & \left(u^{m(x, s)+1}-v^{m(x, s)+1}\right)_{x_{i}} \mathrm{~d} x \mathrm{~d} s \\
= & 0 .
\end{aligned}
$$

In the third place, for the first term on the left-hand side of (47), by Lemma 2 ,

$$
\begin{aligned}
& \lim _{n \rightarrow \infty} \int_{0}^{t} \int_{\Omega} g_{n}\left(u^{m(x, s)+1}-v^{m(x, s)+1}\right) \frac{\partial(u-v)}{\partial t} \mathrm{~d} x \mathrm{~d} s \\
& \quad=\int_{0}^{t} \int_{\Omega} \operatorname{sgn}\left(u^{m(x, s)+1}-v^{m(x, s)+1}\right) \frac{\partial(u-v)}{\partial t} \mathrm{~d} x \mathrm{~d} s \\
& =\int_{0}^{t} \int_{\Omega} \operatorname{sgn}(u-v) \frac{\partial(u-v)}{\partial s} \mathrm{~d} s \\
& =\int_{0}^{t} \frac{d}{d t}\left\|u-v_{1}\right\| \mathrm{d} s .
\end{aligned}
$$




$$
\int_{\Omega}|u(x, t)-v(x, t)| \mathrm{d} x \leq \int_{\Omega}\left|u_{0}(x)-v_{0}(x)\right| \mathrm{d} x .
$$

Corollary 1. Theorem 2 is true.

\section{The Stability Based on the Partial Boundary Value Condition}

Theorem 5. Let $u(x, t)$ and $v(x, t)$ be two solutions of equation (1) with the initial values $u_{0}(x)$ and $v_{0}(x)$ respectively, and with a partial boundary value condition

$$
u(x, t)=v(x, t)=0, \quad(x, t) \in \Sigma_{1} \cup \Sigma_{2},
$$

where

$$
\Sigma_{2}=\{x \in \partial \Omega: a(x, t) \neq 0\},
$$

and for any given $i \in\{1,2, \ldots, N\}$, if $b_{i}^{\prime}(s) \geq 0$,

$$
\Sigma_{1}=\left\{\bigcup_{i=1}^{N} \Sigma_{1 i t} \times(0, T)\right\},
$$

and if $b_{i}^{\prime}(s) \leq 0$,

$$
\Sigma_{1}=\left\{\bigcup_{i=1}^{N} \Sigma_{2 i t} \times(0, T)\right\} .
$$

It is supposed that

$$
\frac{1}{\lambda}\left(\int_{\Omega \backslash \Omega_{\lambda t}} a(x, t)|\nabla a|^{2} d x\right)^{1 / 2} \leq c(T),
$$

and $u(x, t)$ and $v(x, t)$ satisfy

$$
\begin{aligned}
& \int_{\Omega} a(x, t)[1+(m(x, t)+1) \log u]^{2}|\nabla m|^{2} \mathrm{~d} x \leq c(T), \\
& \int_{\Omega} a(x, t)[1+(m(x, t)+1) \log v]^{2}|\nabla m|^{2} \mathrm{~d} x \leq c(T) .
\end{aligned}
$$

Then,

$$
\int_{\Omega}|u(x, t)-v(x, t)| \mathrm{d} x \leq c(T) \int_{\Omega}\left|u_{0}(x)-v_{0}(x)\right| \mathrm{d} x .
$$

Proof. According to the definition of weak solutions, for all $0 \leq \varphi \in C_{0}^{1}\left(Q_{T}\right)$, we have

$$
\begin{aligned}
& \iint_{Q_{T}} u_{t} \varphi(x, t) \mathrm{d} x \mathrm{~d} t+\iint_{Q_{T}} \frac{a(x, t)}{m(x, t)+1} \nabla u^{m(x, t)+1} \nabla \varphi \mathrm{d} x \mathrm{~d} t- \\
& \quad-\iint_{Q_{T}} \frac{a(x, t)}{m(x, t)+1} u^{m(x, t)+1} \log u \nabla m \nabla \varphi \mathrm{d} x \mathrm{~d} t \\
& \quad+\sum_{i=1}^{N} \iint_{Q_{T}} b_{i}\left(u^{m(x, t)+1}\right) \varphi_{x_{i}} \mathrm{~d} x \mathrm{~d} t=0 .
\end{aligned}
$$

For any $t \in[0, T)$ and a small positive constant $\lambda>0$, based on

$$
\Omega_{\lambda t}=\{x \in \Omega: a(x, t)>\lambda\},
$$

we define that

$$
\phi_{\lambda t}(x)= \begin{cases}1, & \text { if } x \in \Omega_{\lambda t}, \\ \frac{a(x, t)}{\lambda}, x \in \Omega \backslash \Omega_{\lambda t} . & \end{cases}
$$
Because

$$
u(x, t)=0=v(x, t), x \in\{x \in \Omega: a(x, t) \neq 0\},
$$

we can choose

$$
\chi_{\tau, s}(t) \phi_{\lambda t}(x) g_{n}\left(u^{m(x, t)+1}-v^{m(x, t)+1}\right),
$$

as the test function. Then,

$$
\begin{aligned}
& \int_{\tau}^{s} \int_{\Omega} \phi_{\lambda t}(x) g_{n}\left(u^{m(x, t)+1}-v^{m(x, t)+1}\right) \frac{\partial(u-v)}{\partial t} \mathrm{~d} x \mathrm{~d} t \\
& \quad+\int_{\tau}^{s} \int_{\Omega} \frac{a(x, t)}{m(x, t)+1}\left(\nabla u^{m(x, t)+1}-\nabla v^{m(x, t)+1}\right) \cdot \phi_{\lambda t}(x) \nabla\left(u^{m(x, t)+1}-v^{m(x, t)+1}\right) \cdot h_{n}\left(u^{m(x, t)+1}-v^{m(x, t)+1}\right) \mathrm{d} x \mathrm{~d} t \\
& \quad+\int_{\tau}^{s} \int_{\Omega} \frac{a(x, t)}{m(x, t)+1}\left(\nabla u^{m(x, t)+1}-\nabla v^{m(x, t)+1}\right) \cdot \nabla \phi_{\lambda}(x, t) g_{n}\left(u^{m(x, t)+1}-v^{m(x, t)+1}\right) \mathrm{d} x \mathrm{~d} t \\
& \quad-\int_{\tau}^{s} \int_{\Omega} \frac{a(x, t)}{m(x, t)+1}\left[u^{m(x, t)+1} \log u-v^{m(x, t)+1} \log v\right] \nabla m \cdot \phi_{\lambda t}(x) \nabla\left(u^{m(x, t)+1}-v^{m(x, t)+1}\right) \cdot h_{n}\left(u^{m(x, t)+1}-v^{m(x, t)+1}\right) \mathrm{d} x \mathrm{~d} t \\
& \quad-\int_{\tau}^{s} \int_{\Omega} \frac{a(x, t)}{m(x, t)+1}\left[u^{m(x, t)+1} \log u-v^{m(x, t)+1} \log v\right] \nabla m \cdot \nabla \phi_{\lambda t}(x) g_{n}\left(u^{m(x, t)+1}-v^{m(x, t)+1}\right) \mathrm{d} x \mathrm{~d} t \\
& \quad+\sum_{i=1}^{N} \int_{\tau}^{s} \int_{\Omega}\left[b_{i}\left(u^{m(x, t)+1}\right)-b_{i}\left(v^{m(x, t)+1}\right)\right]\left[\phi_{\lambda x_{i}}(x) g_{n}\left(u^{m(x, t)+1}-v^{m(x, t)+1}\right)+\phi_{\lambda t}\left(u^{m(x, t)+1}-v^{m(x, t)+1}\right)_{x_{i}} h_{n}\left(u^{m(x, t)+1}-v^{m(x, t)+1}\right)\right] \mathrm{d} x \mathrm{~d} t \\
& =0 .
\end{aligned}
$$


Firstly, we still have

$$
\begin{aligned}
& \int_{\tau}^{s} \int_{\Omega} \frac{a(x, t)}{m(x, t)+1}\left(\nabla u^{m(x, t)+1}-\nabla v^{m(x, t)+1}\right) \\
& \quad \cdot \phi_{\lambda t} \nabla\left(u^{m(x, t)+1}-v^{m(x, t)+1}\right) h_{n}\left(u^{m(x, t)+1}-v^{m(x, t)+1}\right) \mathrm{d} x \mathrm{~d} t
\end{aligned}
$$

$\geq 0$.
Secondly, for the third term on the left-hand side of (71) by that $1 / \lambda\left(\int_{\Omega \backslash \Omega_{\lambda t}} a(x, t)|\nabla a|^{2} \mathrm{~d} x\right)^{1 / 2} \leq c(T)$, we have

$$
\begin{aligned}
& \left|\int_{\tau}^{s} \int_{\Omega} \frac{a(x, t)}{m(x, t)+1}\left(\nabla u^{m(x, t)+1}-\nabla v^{m(x, t)+1}\right) \cdot \nabla \phi_{\lambda t} g_{n}\left(u^{m(x, t)+1}-v^{m(x, t)+1}\right) \mathrm{d} x \mathrm{~d} t\right| \\
& \quad \leq \int_{\tau}^{s} \int_{\Omega \backslash \Omega_{\lambda t}} \frac{a(x, t)}{m(x, t)+1}\left(\nabla u^{m(x, t)+1}-\nabla v^{m(x, t)+1}\right) \cdot \nabla \phi_{\lambda t} g_{n}\left(u^{m(x, t)+1}-v^{m(x, t)+1}\right) \mid \mathrm{d} x \mathrm{~d} t \\
& \quad \leq \int_{\tau}^{s} \int_{\Omega \backslash \Omega_{\lambda t}} \frac{a(x, t)}{m(x, t)+1}\left(\left|\nabla u^{m(x, t)+1}\right|+\left|\nabla v^{m(x, t)+1}\right|\right)\left|\nabla \phi_{\lambda t}\right| \mathrm{d} x \mathrm{~d} t \\
& \quad \leq \int_{\tau}^{s} \frac{1}{\lambda} \int_{\Omega \backslash \Omega_{\lambda t}} \frac{a(x, t)}{m(x, t)+1}\left(\left|\nabla u^{m(x, t)+1}\right|+\left|\nabla v^{(m(x, t)+1}\right|\right)|\nabla a| \mathrm{d} x \mathrm{~d} t \\
& \quad \leq c \int_{\tau}^{s}\left(\int_{\Omega \backslash \Omega_{\lambda t}} a(x, t)\left(\left|\nabla u^{m(x, t)+1}\right|^{2}+\left|\nabla v^{m(x, t)+1}\right|^{2}\right) \mathrm{d} x\right)^{1 / 2} \\
& \quad \cdot \frac{1}{\lambda}\left(\int_{\Omega \backslash \Omega_{\lambda t}} a(x, t)|\nabla a|^{2} \mathrm{~d} x\right)^{1 / 2} \mathrm{~d} t \\
& \quad \longrightarrow 0
\end{aligned}
$$

as $\lambda \longrightarrow 0$. Thirdly, for the fourth term on the left-hand side of (71), we can show

$$
\begin{aligned}
& \mid \int_{\tau}^{s} \int_{\Omega} \frac{a(x, t)}{m(x)+1}\left[u^{m(x, t)+1} \log u-v^{m(x, t)+1} \log v\right] \nabla m \\
& \cdot \phi_{\lambda t}(x) \nabla\left(u^{m(x, t)+1}-v^{m(x, t)+1}\right) h_{n}\left(u^{m(x, t)+1}-v^{m(x, t)+1}\right) \mathrm{d} x \mathrm{~d} t \mid \\
& \leq \int_{\tau}^{s}\left(\int_{D_{n t}} a(x, t)\left|\nabla u^{m(x, t)+1}-\nabla v^{m(x, t)+1}\right|^{2} d x\right)^{1 / 2} \\
& \quad \cdot c\left(\int_{D_{n t}} a(x, t)\left[u^{m(x, t)+1} \log u^{m(x, t)+1}-v^{m(x, t)+1} \log v^{m(x, t)+1}\right]^{2} h_{n}\left(u^{m(x, t)+1}-v^{m(x, t)+1}\right)^{2}|\nabla m|^{2} d x\right)^{1 / 2} \mathrm{~d} t \\
& \quad 0
\end{aligned}
$$

as $\lambda \longrightarrow 0$. The corresponding details are given below.

If $D_{0 t}=\{x \in \Omega:|u-v|=0\}$ is with 0 measure, then 


$$
\begin{aligned}
& \lim _{n \longrightarrow 0} \int_{\tau}^{s} \int_{D_{n t}} a(x, t)\left|\nabla u^{m(x, t)+1}-\nabla v^{m(x, t)+1}\right|^{2} \mathrm{~d} x \mathrm{~d} t \\
& \quad \leq \int_{\tau}^{s} \int_{D_{0 t}} a(x, t)\left(\left|\nabla u^{m(x, t)+1}\right|^{2}+\left|\nabla v^{m(x, t)+1}\right|^{2}\right) \mathrm{d} x \mathrm{~d} t \\
& \quad=0
\end{aligned}
$$

and by (64),

$$
\int_{\Omega} a(x, t)[1+(m(x, t)+1) \log u]^{2}|\nabla m|^{2} \mathrm{~d} x \leq c(T),
$$

$$
\begin{aligned}
& \lim _{n \longrightarrow 0} \int_{\tau}^{s} \int_{D_{n t}} a(x, t)\left[u^{m(x, t)+1} \log u^{m(x, t)+1}-v^{m(x, t)+1} \log v^{m(x, t)+1}\right]^{2} \\
& \cdot h_{n}\left(u^{m(x, t)+1}-v^{m(x, t)+1}\right)^{2}|\nabla m|^{2} \mathrm{~d} x \mathrm{~d} t \mid \\
& \leq \lim _{n \longrightarrow 0} \int_{\tau}^{s} \int_{D_{n t}} a(x, t) \frac{\left[u^{m(x, t)+1} \log u^{m(x, t)+1}-v^{m(x, t)+1} \log v^{m(x, t)+1}\right]^{2}}{\left|u^{m(x, t)+1}-v^{m(x, t)+1}\right|^{2}}|\nabla m|^{2} \mathrm{~d} x \mathrm{~d} t \\
& \leq \int_{\tau}^{s} \int_{D_{0 t}} a(x, t)[1+(m(x, t)+1) \log v]^{2}|\nabla m|^{2} \mathrm{~d} x \\
& \leq c(T) \text {. }
\end{aligned}
$$

When $D_{0 t}=\{x \in \Omega:|u-v|=0\}$ has a positive measure,

Also by (64),

$$
\begin{aligned}
& \lim _{n \rightarrow 0} \int_{\tau}^{s} \int_{D_{n t}} a(x, t)\left|\nabla u^{m(x, t)+1}-\nabla v^{m(x, t)+1}\right|^{2} \mathrm{~d} x \mathrm{~d} t \\
& \quad \leq \int_{\tau}^{s} \int_{\Omega} a(x, t)\left(\left|\nabla u^{m(x, t)+1}\right|^{2}+\left|\nabla v^{m(x, t)+1}\right|^{2}\right) \mathrm{d} x \mathrm{~d} t \\
& \quad \leq c(T) .
\end{aligned}
$$

$$
\begin{aligned}
& \left.\lim _{n \longrightarrow 0} \int_{\tau}^{s}\left|\int_{D_{n t}} a(x, t)\left[u^{m(x, t)+1} \log u^{m(x, t)+1}-v^{m(x, t)+1} \log v^{m(x, t)+1}\right]^{2} h_{n}\left(u^{m(x, t)+1}-v^{m(x, t)+1}\right)^{2}\right| \nabla m\right|^{2} \mathrm{~d} x \mathrm{~d} t \mid \\
& \quad \leq \lim _{n \longrightarrow 0} \int_{\tau}^{s} \int_{D_{0 t}} a(x, t) \frac{\left[u^{m(x, t)+1} \log u^{m(x, t)+1}-v^{m(x, t)+1} \log v^{m(x, t)+1}\right]^{2}}{\left|u^{m(x, t)+1}-v^{m(x, t)+1}\right|^{2}}|\nabla m|^{2} \mathrm{~d} x \mathrm{~d} t \\
& \quad=0 .
\end{aligned}
$$

Fourthly, when $b_{i}^{\prime}(s) \geq 0$, using the partial boundary value condition (59), 


$$
\begin{aligned}
& -\lim _{\lambda \longrightarrow 0} \int_{\tau}^{s} \lim _{n \longrightarrow \infty} \int_{\Omega}\left[b_{i}\left(u^{m(x, t)+1}\right)-b_{i}\left(v^{m(x, t)+1}\right)\right] \phi_{\lambda t x_{i}}(x) g_{n}\left(u^{m(x, t)+1}-v^{m(x, t)+1}\right) \mathrm{d} x \mathrm{~d} t \\
& =-\lim _{\lambda \longrightarrow 0} \int_{\tau}^{s} \int_{\Omega}\left[b_{i}\left(u^{m(x, t)+1}\right)-b_{i}\left(v^{m(x, t)+1}\right)\right] \phi_{\lambda t x_{i}}(x) \operatorname{sign}\left(u^{m(x, t)+1}-v^{m(x, t)+1}\right) \mathrm{d} x \mathrm{~d} t \\
& =-\lim _{\lambda \longrightarrow 0} \int_{\tau}^{s} \int_{\Omega}\left[b_{i}\left(u^{m(x, t)+1}\right)-b_{i}\left(v^{m(x, t)+1}\right)\right] \phi_{\lambda t x_{i}}(x) \operatorname{sign}\left(b_{i}\left(u^{m(x, t)+1}\right)-b_{i}\left(v^{m(x, t)+1}\right)\right) \mathrm{d} x \mathrm{~d} t \\
& =-\lim _{\lambda \longrightarrow 0} \int_{\tau}^{s} \int_{\Omega \backslash \Omega_{\lambda t}}\left|b_{i}\left(u^{m(x, t)+1}\right)-b_{i}\left(v^{m(x, t)+1}\right)\right| \frac{a_{x_{i}}}{\lambda} \mathrm{d} x \mathrm{~d} t \\
& =-\lim _{\lambda \longrightarrow 0} \int_{\tau}^{s} \int_{\Omega \backslash \Omega_{\lambda t}}\left|u^{m(x, t)+1}-v^{m(x, t)+1}\right| b_{i}^{\prime}(\xi) \frac{a_{x_{i}}}{\lambda} \mathrm{d} x \mathrm{~d} t \\
& \leq \lim _{\lambda \longrightarrow 0} \int_{\tau}^{s} \frac{1}{\lambda} \int_{\Omega_{\lambda i t}}\left(-c_{i} a_{x_{i}}\right)\left|u^{m(x, t)+1}-v^{m(x, t)+1}\right| \mathrm{d} x \mathrm{~d} t \\
& \quad=\int_{\tau}^{s} \int_{\Sigma_{i 1 t}}\left(-c_{i} a_{x_{i}}\right)\left|u^{m(x, t)+1}-v^{m(x, t)+1}\right| \mathrm{d} \Sigma \mathrm{d} t \\
& =0 .
\end{aligned}
$$

When $b_{i}^{\prime}(s) \leq 0$, using the partial boundary value condition (59), we have

$$
\begin{aligned}
& -\lim _{\lambda \longrightarrow 0} \lim _{n \longrightarrow \infty} \int_{\tau}^{s} \int_{\Omega}\left[b_{i}\left(u^{m(x, t)+1}\right)-b_{i}\left(v^{m(x, t)+1}\right)\right] \phi_{\lambda t x_{i}}(x) g_{n}\left(u^{m(x, t)+1}-v^{m(x, t)+1}\right) \mathrm{d} x \mathrm{~d} t \\
& =-\lim _{\lambda \longrightarrow 0} \int_{\tau}^{s} \int_{\Omega}\left[b_{i}\left(u^{m(x, t)+1}\right)-b_{i}\left(v^{m(x, t)+1}\right)\right] \phi_{\lambda t x_{i}}(x) \operatorname{sign}\left(u^{m(x, t)+1}-v^{m(x, t)+1}\right) \mathrm{d} x \mathrm{~d} t \\
& =-\lim _{\lambda \longrightarrow 0} \int_{\tau}^{s} \int_{\Omega}\left[b_{i}\left(u^{m(x, t)+1}\right)-b_{i}\left(v^{m(x, t)+1}\right)\right] \phi_{\lambda t x_{i}}(x) \operatorname{sign}\left(b_{i}\left(u^{m(x, t)+1}\right)-b_{i}\left(v^{m(x, t)+1}\right)\right) \mathrm{d} x \mathrm{~d} t \\
& =-\lim _{\lambda \longrightarrow 0} \int_{\tau}^{s} \int_{\Omega \backslash \Omega_{\lambda t}}\left|b_{i}\left(u^{m(x, t)+1}\right)-b_{i}\left(v^{m(x, t)+1}\right)\right| \frac{a_{x_{i}}}{\lambda} \mathrm{d} x \mathrm{~d} t \\
& =-\lim _{\lambda \longrightarrow 0} \int_{\tau}^{s} \int_{\Omega \backslash \Omega_{\lambda t}}\left|u^{m(x, t)+1}-v^{m(x, t)+1}\right| b_{i}(\xi) \frac{a_{x_{i}}}{\lambda} \mathrm{d} x \mathrm{~d} t \\
& \leq \lim _{\lambda \longrightarrow 0} \int_{\tau}^{s} \frac{1}{\lambda} \int_{\Omega_{\lambda i 2 t}}\left(-c_{i} a_{x_{i}}\right)\left|u^{m(x, t)+1}-v^{m(x, t)+1}\right| \mathrm{d} x \mathrm{~d} t \\
& \quad=\int_{\tau}^{s} \int_{\Sigma_{i 2 t}}\left(-c_{i} a_{x_{i}}\right)\left|u^{m(x, t)+1}-v^{m(x, t)+1}\right| \mathrm{d} \Sigma \mathrm{d} t \\
& \quad=0 .
\end{aligned}
$$

The last but not the least, as in the proof of Theorem 2, we can show that 


$$
\begin{aligned}
\lim _{\lambda \longrightarrow 0} & \lim _{n \longrightarrow \infty} \int_{\tau}^{s} \int_{\Omega}\left[b_{i}\left(u^{m(x, t)+1}\right)-b_{i}\left(v^{m(x, t)+1}\right)\right] \phi_{\lambda t}\left(u^{m(x, t)+1}-v^{m(x, t)+1}\right)_{x_{i}} \\
& \cdot h_{n}\left(u^{m(x, t)+1}-v^{m(x, t)+1}\right) \mathrm{d} x \mathrm{~d} t \\
& =0 .
\end{aligned}
$$

$$
\begin{aligned}
& \text { Then, } \\
& \lim _{n \longrightarrow \infty} \lim _{\lambda \longrightarrow 0} \int_{\tau}^{s} \int_{\Omega} g_{n}\left(u^{m(x, t)+1}-v^{m(x, t)+1}\right) \phi_{\lambda t} \frac{\partial(u-v)}{\partial t} \mathrm{~d} x \mathrm{~d} t \\
& =\int_{\Omega}|u(x, s)-v(x, s)| \mathrm{d} x-\int_{\Omega}|u(x, \tau)-v(x, \tau)| \mathrm{d} x .
\end{aligned}
$$

Letting $\lambda \longrightarrow 0$ and $n \longrightarrow \infty$ in (73), by (74) and (80)-(85), we obtain

$$
\int_{\Omega}|u(x, s)-v(x, s)| \mathrm{d} x \leq c \int_{\Omega}|u(x, \tau)-v(x, \tau)| \mathrm{d} x .
$$

By the arbitraries of $\tau$, we have

$$
\int_{\Omega}|u(x, t)-v(x, t)| \mathrm{d} x \leq \int_{\Omega}\left|u_{0}(x)-v_{0}(x)\right| \mathrm{d} x .
$$

Proof of Theorem 2. Because for every $i \in\{1,2, \ldots, N\}$, either $b_{i}^{\prime}(s) \geq 0$ or $b_{i}^{\prime}(s) \leq 0$, by checking the process of the proof of (80) or (81), we easily obtain Theorem 3.

\section{Conclusion}

In this paper, we consider the initial-boundary value problem of a generalized porous medium equation with a variable exponent. Different from the previous related works, both the diffusion coefficient $a(x, t)$ and variable exponent $p(x, t)$ are dependent on the time variable $t$, we find out a partial boundary value condition matching up with the equation. The most important innovation is that the partial boundary value condition matching up with the equation is based on a submanifold of $\partial \Omega \times(0, T)$. However, because there is an additional condition (23) imposed, Theorem 3 has not answered the problem globally. In other words, how to obtain the same conclusion as that in Theorem 3 without condition (23) is remained to be studied in the future.

\section{Data Availability}

No data were used to support this study.

\section{Conflicts of Interest}

The author declares that he has no conflicts of interest.

\section{Acknowledgments}

This study was supported by the Natural Science Foundation of Fujian Province (2019J01858), China.

\section{References}

[1] A. Friedman, Partial Differential Equations of Parabolic Type, Prentice-Hall, Englewood Cliffs, NJ, USA, 1964.

[2] J. L. Vazquez, Smoothing and Decay Estimates for Nonlinear Diffusion Equations, Equations of Porous Medium Type, Oxford University Press, Oxford, UK, 2006.

[3] J. Smoller, Shock Waves and Reaction Diffusion Equations, Academic Press, New York, NY, USA, 1983.

[4] A. S. Alexander, A. G. Victor, P. K. Sergei, and P. M. Alexander, Blow-up in Quasilinear Parabolic Equations, Walter de Gruyter, Berlin, Germany, 1995.

[5] Z. Wu, J. Zhao, J. Yin, and H. Li, Nonlinear Diffusion Equations, Word Scientific Publishing, Singapore, 2001.

[6] E. DiBenedetto, Degenerate Parabolic Equations, SpringerVerlag, New York, NY, USA, 1993.

[7] Y.-X. Chen, "Excitation manipulation of three-dimensional completely localized rogue waves in a partially nonlocal and inhomogeneous nonlinear medium," Nonlinear Dynamics, vol. 97, no. 1, pp. 177-184, 2019.

[8] C.-Q. Dai, Y. Fan, and Y.-Y. Wang, "Three-dimensional optical solitons formed by the balance between different-order nonlinearities and high-order dispersion/diffraction in paritytime symmetric potentials," Nonlinear Dynamics, vol. 98, no. 1, pp. 489-499, 2019.

[9] C.-Q. Dai, Y.-Y. Wang, Y. Fan, and J.-F. Zhang, "Interactions between exotic multi-valued solitons of the $(2+1)$-dimensional Korteweg-de Vries equation describing shallow water wave," Applied Mathematical Modelling, vol. 80, pp. 506-515, 2020.

[10] S. N. Antontsev and S. I. Shmarev, "A model porous medium equation with variable exponent of nonlinearity: existence, uniqueness and localization properties of solutions," Nonlinear Analysis: Theory, Methods \& Applications, vol. 60, no. 3, pp. 515-545, 2005.

[11] J. C. M. Duque, R. M. P. Almeida, and S. N. Antontsev, "Convergence of the finite element method for the porous media equation with variable exponent," SIAM Journal on Numerical Analysis, vol. 51, no. 6, pp. 3483-3504, 2013.

[12] J. C. M. Duque, R. M. P. Almeida, and S. N. Antontsev, "Numerical study of the porous medium equation with absorption, variable exponents of nonlinearity and free boundary," Applied Mathematics and Computation, vol. 235, pp. 137-147, 2014.

[13] W. Huang and R. D. Russell, "Moving mesh strategy based on a gradient flow equation for two-dimensional problems," SIAM Journal on Scientific Computing, vol. 20, no. 3, pp. 998-1015, 1998.

[14] Y. Li and Q. Wang, "Homogeneous Dirichlet problems for quasilinear anisotropic degenerate parabolic-hyperbolic equations," Journal of Differential Equations, vol. 252, no. 9, pp. 4719-4741, 2012.

[15] P.-L. Lions, B. Perthame, and E. Tadmor, "A kinetic formulation of multidimensional scalar conservation laws and related equations," Journal of the American Mathematical Society, vol. 7, no. 1, p. 169, 1994. 
[16] F. Antonelli, E. Barucci, and M. E. Mancino, "A comparison result for FBSDE with applications to decisions theory," Mathematical Methods of Operations Research, vol. 54, no. 3, pp. 407-423, 2001.

[17] C. Bardos, A. Y. Leroux, and J. C. Nedelec, "First order quasilinear equations with boundary conditions," Communications in Partial Differential Equations, vol. 4, no. 9, pp. 1017-1034, 1979.

[18] B. Andreianov, M. Bendahmane, K. H. Karlsen, and S. Ouaro, "Well-posedness results for triply nonlinear degenerate parabolic equations," Journal of Differential Equations, vol. 247, no. 1, pp. 277-302, 2009.

[19] K. Kobayasi and H. Ohwa, "Uniqueness and existence for anisotropic degenerate parabolic equations with boundary conditions on a bounded rectangle," Journal of Differential Equations, vol. 252, no. 1, pp. 137-167, 2012.

[20] M. Ruzicka, "Electrorheological fluids: modeling and mathematical theory," in Lecture Notes in Mathematics, Vol. 1748, Springer, Berlin, Germany, 2000.

[21] E. Acerbi and G. Mingione, "Regularity results for stationary electro-rheological fluids," Archive for Rational Mechanics and Analysis, vol. 164, no. 3, pp. 213-259, 2002.

[22] S. Antontsev and S. Shmarev, "Parabolic equations with double variable nonlinearities," Mathematics and Computers in Simulation, vol. 81, no. 10, pp. 2018-2032, 2011.

[23] S. Lian, W. Gao, H. Yuan, and C. Cao, "Existence of solutions to an initial Dirichlet problem of evolutional $p(x)$-Laplace equations," Annales de l'Institut Henri Poincare (C) Non Linear Analysis, vol. 29, no. 3, pp. 377-399, 2012.

[24] J. Aramaki, "Hölder continuity with exponent $(1+\alpha) / 2$ in the time variable for solutions of parabolic equations," Electronic Journal of Differential Equations, vol. 96, pp. 1-6, 2015.

[25] D.-J. Ding, D.-Q. Jin, and C.-Q. Dai, "Analytical solutions of differential-difference sine-Gordon equation," Thermal Science, vol. 21, no. 4, pp. 1701-1705, 2017.

[26] N. Zhu, C. Pan, and Z. Liu, "Re-study on localized structures based on variable separation solutions from the modified tanh-function method," Nonlinear Dynamics, vol. 83, pp. 1331-1339, 2016.

[27] Y.-Y. Wang, L. Chen, C.-Q. Dai, J. Zheng, and Y. Fan, "Exact vector multipole and vortex solitons in the media with spatially modulated cubic-quintic nonlinearity," Nonlinear Dynamics, vol. 90, no. 2, pp. 1269-1275, 2017.

[28] H. Zhan and Z. Feng, "Well-posedness problem of an anisotropic parabolic equation," Journal of Differential Equations, vol. 268, no. 2, pp. 389-413, 2020.

[29] H. Zhan, "Evolutionary $p(x)$-Laplacian equation with a convection term," Acta Mathematicae Applicatae Sinica, English Series, vol. 35, no. 3, pp. 655-670, 2019.

[30] H. Zhan, "The weak solutions of an evolutionary $p(x)$-Laplacian equation are controlled by the initial value," Computers \& Mathematics with Applications, vol. 76, no. 9, pp. 22722285, 2018.

[31] H. Zhan and Z. Feng, "Solutions of evolutionary $p(x)$-Laplacian equation based on the weighted variable exponent space," Zeitschrift für angewandte Mathematik und Physik, vol. 68, no. 6, p. 134, 2017.

[32] H. Zhan, "On the solutions of a porous medium equation with exponent variable," Discrete Dynamics in Nature and Society, vol. 2019, Article ID 9290582, 15 pages, 2019. 